\title{
Für einen guten Start mit Insulin
}

\author{
Ein neues Schulungsprogramm soll Patienten mit Diabetes Typ 2, die eine \\ Insulintherapie benötigen, den Behandlungsbeginn erleichtern.
}

„Eine strukturierte Schulung zu Anfang der Therapie ist nicht die Regel“, sagte Dr. Stephan Kress vom Vinzentius-Krankenhaus in Landau. Viele Patienten seien mit ihren Sorgen in den ersten Wochen auf sich allein gestellt, da im Praxisalltag für Schulungen nur wenig Zeit bleibe. Mit dem Schulungsprogramm „bot leben“ haben Fachärzte, Diabetesberater und der Insulinhersteller Sanofi Diabetes sektorenübergreifende Lehreinheiten erarbeitet. In vier 60-minütigen Modulen lernen Patienten, wie sie Blutzucker messen, Insulin spritzen und Unterzuckerung vorbeugen können. Trainer können neben Ärzten aus Schwerpunktpraxen sowohl Ärzte und Krankenschwestern aus der Klinik als auch qualifizierte Hausärzte, medizinische Fachangestellte oder Dia- betesberater sein. Sie sprechen bei den Sitzungen auch Ängste und Wünsche der Patienten an.

Das erste Modul ist ein Einzelgespräch in der Klinik, beim Hausarzt oder in der Schwerpunktpraxis. Die Module zwei bis vier sind Gruppenschulungen. Ideal seien Gruppen mit vier bis sechs Patienten, berichtete Diabetesberaterin Helga Varlemann aus Hamburg. Die erworbenen Kenntnisse und der Umgang mit Blutzuckermessgerät oder die Insulingabe werden zu Beginn jeder Einheit abgefragt. Dabei können Ärzte Wissenslücken der Patienten feststellen und nachschulen. Auch für die Lebensführung erhalten $\mathrm{Pa}$ tienten Tipps, etwa zu Ernährung oder Bewegung. Die Lerneinheiten zwei bis vier behandeln je ein Schwerpunktthe- ma: „Fit für die Insulingabe“, „Unterzuckerung erkennen und vermeiden“" sowie „Im Alltag zurechtkommen“. Die einzelnen Schulungsschritte werden in einem Insulin-Führerschein für Patienten und Ärzte dokumentiert.

Damit Ärzte und Medizinische Fachangestellte in ihrer Praxis oder Klinik mit „bot leben“ lehren können, müssen sie ein eineinhalbtägiges Train-the-Trainer-Seminar besuchen. Dieses ist nicht in den 229 Euro für das Schulungsmaterial enthalten und wird über Sanofi organisiert. Seit April können Interessierte den Schulungskoffer - inklusive Curriculum, Folienordner, Tischflipchart, Tagebuch, Insulin-Führerschein, Patienten-Fibel, Kopiervorlagen - und Verbrauchsmaterial beim Kirchheim Verlag bestellen. Ab 2014 rechnen die Initiatoren mit der Freigabe durch das Bundesversicherungsamt, sodass das Schulungsprogramm dann auch abgerechnet werden kann.

Johanna Dielmann-von Berg

Quelle: Veranstaltung zur Vorstellung des Programms „bot leben“ am 27.3.13 in Berlin, auf Einladung von Sanofi

\section{Aktiv für ältere Patienten}

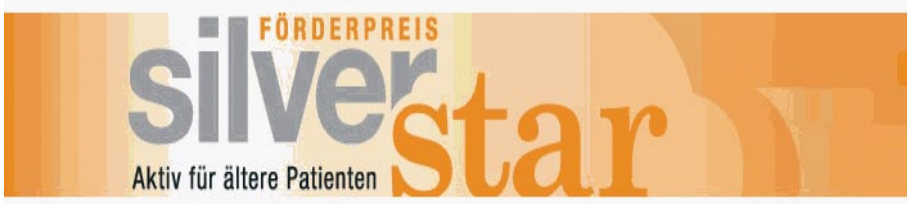

In Würde altern, das ist es, was sich jeder Mensch wünscht. Gesundheit und ein selbstbestimmtes Leben gehören dazu. Dass ein erfülltes Leben auch für ältere Menschen mit Diabetes möglich bleibt, ist das Ziel des SilverStar - dem Förderpreis von Berlin-Chemie.

Der SilverStar ist ein Preis, mit dem Berlin-Chemie Projekte oder Initiativen fördert, die zu einer besseren Versorgung älterer Menschen mit Diabetes beitragen. Getreu dem Motto „Aktiv für ältere Patienten" kann sich jeder um den SilverStar bewerben - ob Gesundheitsprofis wie Ärzte, Pflegekräfte, Apotheker oder engagierte Angehörige, Freunde, Nachbarn oder selbst Betroffene: Gesucht werden kreative Ideen, die älteren Menschen mit Diabetes den Umgang mit ihrer Erkrankung erleichtern, heißt es auf der Website zum SilverStar.

SilverStar - der Name ist Programm Der SilverStar steht symbolisch für die Generation der "Silberhaarigen“, das heißt für ältere Menschen mit Diabetes.
Sie sind die Stars, sie sollen im Mittelpunkt stehen.

Diabetes kann für viele ältere Menschen zum Problem werden, denn er kann die Lebensqualität beeinträchtigen. Der SilverStar will zeigen, dass es sich lohnt, den Kopf nicht in den Sand zu stecken. Wer aktiv bleibt und positiv denkt, kann viel erreichen. Das gilt nicht nur für die Betroffenen selbst, sondern auch für die Menschen im sozialen Umfeld, die sich aktiv für ältere Menschen mit Diabetes einsetzen. Dieses Engagement will der SilverStar würdigen und fördern.

\section{Förderpreis soll Zeichen setzen}

Die geriatrische Diabetestherapie wird in den nächsten Jahren weiter an Bedeutung gewinnen und zu einer der größten Her- ausforderungen in der medizinischen Versorgung werden. Bereits jetzt unterstützt das Unternehmen zahlreiche Projekte in den Bereichen Diabetes und Altersmedizin.

Mit dem Förderpreis SilverStar, der 2013 zum dritten Mal verliehen wird, setzt das Unternehmen ein weiteres Zeichen und fördert mit einer Gesamtsumme von 25000 Euro Projekte, die zu einer Verbesserung der Versorgung und zu einer Erhöhung der Lebensqualität älterer Menschen mit Diabetes beitragen.

Die Bewerbungsfrist für 2013 endet am 30. Juni. Infos und Bewerbung unter: www.silverstar-preis.de

Quelle: Website www.silverstar-preis.de 